\title{
Continuous and Discontinuous Piecewise Linear Solutions of the Linearly Forced Inviscid Burgers Equation
}

\author{
Hans Lundmark ${ }^{a}$ and Jacek Szmigielski ${ }^{b}$ \\ ${ }^{a}$ Department of Mathematics, Linköping University, SE-581 83 Linköping, Sweden \\ E-mail: halun@mai.liu.se \\ ${ }^{b}$ Department of Mathematics and Statistics, University of Saskatchewan, 106 Wiggins Road, \\ Saskatoon, Saskatchewan, S7N 5E6, Canada \\ E-mail: szmigiel@math.usask.ca
}

\begin{abstract}
We study a class of piecewise linear solutions to the inviscid Burgers equation driven by a linear forcing term. Inspired by the analogy with peakons, we think of these solutions as being made up of solitons situated at the breakpoints. We derive and solve ODEs governing the soliton dynamics, first for continuous solutions, and then for more general shock wave solutions with discontinuities. We show that triple collisions of solitons cannot take place for continuous solutions, but give an example of a triple collision in the presence of a shock.
\end{abstract}

\section{Introduction}

The subject of this paper is piecewise linear solutions of the PDE

$$
\left(u_{t}+u u_{x}\right)_{x x}=0,
$$

which we earlier [10] have called the derivative Burgers equation. This name refers of course to the well-known Burgers equation $u_{t}+u u_{x}=v u_{x x}$ and its special case the inviscid Burgers equation $u_{t}+u u_{x}=0$, which is the prototype equation for studying shock wave solutions of hyperbolic conservation laws. In some applications one considers also forced Burgers equations with terms of the form $F(x, t)$ on the right-hand side, often written as $F=-\partial V / \partial x$ with a potential $V$. Since equation (1.1) is equivalent to $u_{t}+u u_{x}=A(t) x+B(t)$, it is perhaps more appropriate to talk about it as a forced inviscid Burgers equation with linear force (or quadratic potential). Moreover, the latter equation can be rewritten as

$$
u_{t}+\frac{1}{2}\left(u^{2}\right)_{x}=A(t) x+B(t),
$$

which makes sense for a much larger class of functions than just $u \in C^{1}(\mathbf{R})$. For example, if $u \in L_{\text {loc }}^{2}(\mathbf{R})$ we can interpret (1.2) to hold in the sense of distributions. One could work with distributions acting on test functions $\psi(x, t) \in \mathscr{D}\left(\mathbf{R}^{2}\right)$, but the following simpler interpretation is 
sufficient for our purposes here: we view $u(x, t)$ as a mapping that takes a real number $t$ to a function $u(\cdot, t) \in L_{\mathrm{loc}}^{2}(\mathbf{R})$ which we can identify with a distribution in $\mathscr{D}^{\prime}(\mathbf{R})$. The derivative with respect to $x$ is then the distributional derivative defined by its action on a test function $\psi(x) \in$ $\mathscr{D}(\mathbf{R})$ in the usual way, $\left\langle u_{x}, \psi\right\rangle=-\left\langle u, \psi_{x}\right\rangle$, while the derivative with respect to $t$ is the limit of a difference quotient. If equation (1.2) is satisfied in $\mathscr{D}^{\prime}(\mathbf{R})$ for each $t$, then we then say that it holds in a weak sense and that $u$ is its weak solution.

We were led to the Burgers equation by our previous work on peakon and shockpeakon solution of the Degasperis-Procesi (DP) equation

$$
u_{t}-u_{t x x}+4 u u_{x}=3 u_{x} u_{x x}+u u_{x x x},
$$

an integrable wave equation discovered a few years ago $[6,5]$. Indeed, the problems treated in this paper are to some extent "toy problems", but we hope that they might provide some guidance and intuition for the future study of the DP equation.

Equation (1.1) can be obtained formally from the DP equation by substituting $x \mapsto \varepsilon x, t \mapsto \varepsilon t$, and then letting $\varepsilon \rightarrow 0$. This "high-frequency limit" is a natural thing to try on the DP equation, since it is the same procedure that takes the celebrated integrable Camassa-Holm $(\mathrm{CH})$ shallow water equation [4],

$$
u_{t}-u_{t x x}+3 u u_{x}=2 u_{x} u_{x x}+u u_{x x x},
$$

to the Hunter-Saxton (HS) equation for nematic liquid crystals [8, 9],

$$
\left(u_{t}+u u_{x}\right)_{x x}=u_{x} u_{x x} .
$$

The $\mathrm{CH}$ and DP equations both admit peakon solutions, which are multisoliton solutions of the form

$$
u(x, t)=\sum_{k=1}^{n} m_{k}(t) \exp \left(-\left|x-x_{k}(t)\right|\right),
$$

where the functions $x_{k}(t)$ and $m_{k}(t)$ (positions and momenta of the individual peak-shaped solitons) are required to satisfy a certain system of $2 n$ ODEs in order for $u(x, t)$ to satisfy the PDE in a weak sense. In shorthand notation these ODEs are $\dot{x}_{k}=u\left(x_{k}\right), \dot{m}_{k}=-(b-1) u_{x}\left(x_{k}\right)$, where $b=2$ for the $\mathrm{CH}$ equation and $b=3$ for the DP equation. One can think of this as an integrable mechanical system of $n$ particles on the real line, simililar to, for example, the open Toda lattice. If follows from the rapid decay of $e^{-|x|}$ that $\dot{x}_{k}=u\left(x_{k}\right) \approx m_{k}$ when all distances $\left|x_{i}-x_{j}\right|$ are large, so it agrees with intuition to regard $m_{k}$ as the momentum of the $k$ th particle. Asympotically (when $t \rightarrow \pm \infty$ ) the particles will spread apart, each moving with its own (nearly) constant velocity which is nonzero and distinct from the other particles' velocities. The latter is a highly nontrivial fact for the DP equation [13, Theorem 2.4].

There is an analogous class of solutions of the HS equation and the forced Burgers equation (1.2), namely the piecewise linear solutions

$$
u(x, t)=\sum_{k=1}^{n} m_{k}(t)\left|x-x_{k}(t)\right|
$$

In the shorthand notation used above, the governing ODEs take exactly the same form again: $\dot{x}_{k}=u\left(x_{k}\right), \dot{m}_{k}=-(b-1) u_{x}\left(x_{k}\right)$, where $b=2$ for the HS equation and $b=3$ for the forced 
Burgers equation. However, for peakons the term $m_{k} e^{-\left|x_{k}-x_{k}\right|}$ usually dominates the other terms in the equation $\dot{x}_{k}=u\left(x_{k}\right)$, while here we instead have the term $m_{k}\left|x_{k}-x_{k}\right|$ which is zero while all other terms are large. Thus, in contrast to peakons where the interaction is strongly localized, these piecewise linear solitons influence each other more strongly the more separated they are. Although it is a bit hard to develop a useful intuition about these ODEs as a "mechanical" system (perhaps one can think of some kind of expanding gas with long-range correlations), the analogy with peakons still makes it natural to think of the piecewise linear solutions as being composed of some kind of solitons situated at the breakpoints $x_{k}$. (But we have not been able to make sense of the idea that the piecewise linear solutions are somehow high-frequency limits of peakons).

In all four cases mentioned above, the ODEs governing the soliton dynamics can be explicitly solved using inverse spectral methods [1, 12, 13, 2, 10, 3]. In the forced Burgers case the ODEs are also easily solved directly by elementary methods, as we will see.

In the Degasperis-Procesi equation (but not in the Camassa-Holm equation) there also appears a more complicated phenomenon, namely discontinuous solutions of the form

$$
u(x, t)=\sum_{k=1}^{n}\left(m_{k}(t)-s_{k}(t) \operatorname{sgn}\left(x-x_{k}(t)\right)\right) \exp \left(-\left|x-x_{k}(t)\right|\right) .
$$

Such shockpeakons [11] are governed by $3 n$ ODEs for positions $x_{k}$, momenta $m_{k}$, and shock strengths $s_{k}$. Even if one starts with the usual peakon ansatz (1.6), shock solutions of the form (1.8) can form after finite time when a peakon with $m_{k}>0$ collides with an antipeakon with $m_{k+1}<0$ moving in the opposite direction. (In the $\mathrm{CH}$ equation, such collisions give rise to "zerostrength shocks" where $u_{x}$ momentarily blows up but $u$ remains continuous, still being of the form (1.6) after the collision [1], and a similar thing occurs for the HS equation [9].) The shockpeakon ODEs have so far only been solved in the trivial case $n=1$ and in a very particular subcase when $n=2$. The problem is that the Lax pair for the DP equation, which was crucial for deriving the peakon solution formulas, does not make sense for the weak formulation of the DP equation that is used when working with discontinuous solutions.

The forced Burgers equation (1.2) admits an analogous class of solutions, given by the discontinuous piecewise linear ansatz

$$
u(x, t)=\sum_{k=1}^{n}\left(m_{k}(t)\left|x-x_{k}(t)\right|-s_{k}(t) \operatorname{sgn}\left(x-x_{k}(t)\right)\right) .
$$

Such solutions with shocks can form after finite time, even if the initial profile is continuous. Unlike the Degasperis-Procesi case, it turns out here that the extra generality of having jumps in $u$ can be handled without problems.

The outline of the paper is simple: we derive and solve the ODEs govering piecewise linear solutions of the forced Burgers equation (1.2), first in the simpler case (1.7) of continuous solutions (using elementary methods and, for comparison, inverse spectral methods), then in the general case (1.9) of discontinuous solutions (by reduction to the previous case). We conclude with a few examples. 


\section{Continuous piecewise linear solutions}

Theorem 1. The continuous piecewise linear ansatz (1.7), $u=\sum m_{k}\left|x-x_{k}\right|$, is a weak solution to the linearly forced inviscid Burgers equation (1.2) if and only if

$$
\dot{x}_{k}=\sum_{i=1}^{n} m_{i}\left|x_{k}-x_{i}\right|, \quad \dot{m}_{k}=2 m_{k} \sum_{i=1}^{n} m_{i} \operatorname{sgn}\left(x_{i}-x_{k}\right),
$$

for $k=1, \ldots, n$. For this class of solutions, equation (1.2) takes the form

$$
u_{t}+\frac{1}{2}\left(u^{2}\right)_{x}=M^{2} x-M M_{+},
$$

where $M=\sum_{k=1}^{n} m_{k}$ and $M_{+}=\sum_{k=1}^{n} m_{k} x_{k}$ are constants of motion.

Proof. This is a special case (all $s_{k}=0$ ) of Theorem 7 which is proved later.

One can assume that all $m_{k} \neq 0$, since it follows from (2.1) that any vanishing $m_{k}$ remains identically zero. If we think of $x_{k}$ and $m_{k}$ as positions and masses of particles on a line, then the total mass $M$ and the center of mass $M_{+} / M$ (if $M \neq 0$ ) are conserved. Note that when $M=0$ we have the unforced Burgers equation. There are some additional constants of motion $M_{2}, \ldots, M_{n}$ that come together with $M_{1}=M$ from the Lax pair presented in the next section, but we will not need them here [10].

The presence of absolute values and the sign function in (2.1) naturally divides the position space $\mathbf{R}^{n}$ into sectors. More precisely, to any permutation $\sigma=\sigma_{1} \sigma_{2} \ldots \sigma_{n}$ of the numbers $\{1,2, \ldots, n\}$ one can assign the sector $X_{\sigma}=\left\{\left(x_{1}, x_{2}, \ldots, x_{n}\right) \in \mathbf{R}^{n} \mid x_{\sigma_{1}}<x_{\sigma_{2}}<\cdots<x_{\sigma_{n}}\right\}$. We will concentrate on the sector $X_{e}$ corresponding to the identity permutation $e=12 \ldots n$, since there is no loss of generality in assuming that the initial positions $x_{k}(0)$ are sorted in increasing order:

$$
X_{e}=\left\{\left(x_{1}, x_{2}, \ldots, x_{n}\right) \in \mathbf{R}^{n} \mid x_{1}<x_{2}<\cdots<x_{n}\right\} .
$$

For positions in $X_{e}$ the ODEs (2.1) take the form

$$
\dot{x}_{k}=\sum_{i=1}^{n} m_{i}\left(x_{k}-x_{i}\right) \operatorname{sgn}(k-i), \quad \dot{m}_{k}=2 m_{k} \sum_{i=1}^{n} m_{i} \operatorname{sgn}(i-k) .
$$

The following theorem solves this system completely.

Theorem 2. Given any initial data $\left\{x_{k}(0), m_{k}(0)\right\}_{k=1}^{n}$ (with the $x_{k}(0)$ 's ordered or not), the solution of the ODEs (2.4) is given by the formulas below, where $M=\sum m_{k}$ and $M_{+}=\sum m_{k} x_{k}$ as before, and where the empty sums $\sum_{1}^{0}$ and $\sum_{n+1}^{n}$ in $F_{0}$ and $F_{n}$ are to be interpreted as zero (so that $F_{0}(t)=e^{-M t}$ and $\left.F_{n}(t)=e^{M t}\right)$.

- When $M \neq 0$ the solution of (2.4) is

$$
\begin{aligned}
x_{k}(t)= & \frac{M_{+}}{M}+\frac{e^{M t}}{M}\left(\sum_{j<k}\left(x_{k}(0)-x_{j}(0)\right) m_{j}(0)\right) \\
& +\frac{e^{-M t}}{M}\left(\sum_{j>k}\left(x_{k}(0)-x_{j}(0)\right) m_{j}(0)\right), \\
m_{k}(t)= & \frac{m_{k}(0)}{F_{k-1}(t) F_{k}(t)},
\end{aligned}
$$


for $k=1, \ldots, n$, where

$$
F_{k}(t)=\frac{e^{M t}}{M}\left(\sum_{j=1}^{k} m_{j}(0)\right)+\frac{e^{-M t}}{M}\left(\sum_{j=k+1}^{n} m_{j}(0)\right) .
$$

- When $M=0$ the solution of (2.4) is

$$
\begin{aligned}
& x_{k}(t)=x_{k}(0)+t\left(\sum_{j<k}\left(x_{k}(0)-x_{j}(0)\right) m_{j}(0)-\sum_{j>k}\left(x_{k}(0)-x_{j}(0)\right) m_{j}(0)\right), \\
& m_{k}(t)=\frac{m_{k}(0)}{F_{k-1}(t) F_{k}(t)}
\end{aligned}
$$

for $k=1, \ldots, n$, where

$$
F_{k}(t)=1+t\left(\sum_{j=1}^{k} m_{j}(0)-\sum_{j=k+1}^{n} m_{j}(0)\right) .
$$

- Letting $l_{k}=x_{k+1}-x_{k}$ for $k=1, \ldots, n-1$, we have in both cases

$$
l_{k}(t)=l_{k}(0) F_{k}(t) \text {. }
$$

The proof is presented at the end of this section. As an immediate corollary we obtain information about the original ODEs (2.1).

Theorem 3. Given initial data $\left\{x_{k}(0), m_{k}(0)\right\}_{k=1}^{n}$ to the ODEs (2.1) such that $x_{1}(0)<x_{2}(0)<$ $\cdots<x_{n}(0)$ (that is, with the positions in the sector $X_{e}$ of $\mathbf{R}^{n}$ ), the solution is given locally (around $t=0)$ by the formulas of Theorem 2, and this solution is valid as long as the positions $x_{k}(t)$ remain in $X_{e}$.

A local solution that starts in $X_{e}$ hits the boundary of $X_{e}$ whenever $x_{k}=x_{k+1}$ for at least one $k$, an event which we refer to as a collision. It is clear from (2.9) that a collision occurs when some $F_{k}$ becomes zero, at which time $m_{k}$ and $m_{k+1}$ blow up. The local solution is valid up until the time of the first collision. In general a shock will then form, and the continuous ansatz (1.7) will not be able to describe the solution beyond the point of collision. We will return to this in the section about discontinuous solutions.

If all $m_{k}(0)$ 's have the same sign, then (2.6) shows that there are no collisions, so the solution is global. In the case when all are positive, the asymptotic behaviour of this global solution as $t \rightarrow+\infty$ is that $x_{1} \rightarrow M_{+} / M$ and $m_{1} \rightarrow M$, while $x_{k} \rightarrow+\infty$ and $m_{k} \rightarrow 0$ for all $k>1$. When the $m_{k}(0)$ 's have mixed signs, collisions may or may not occur for $t>0$. For example, in the case $n=2$ a collision takes place when $F_{1}(t)=\left(m_{1}(0) e^{M t}+m_{2}(0) e^{-M t}\right) / M$ becomes zero, which happens when $m_{2}(0) / m_{1}(0)<0$ and $t=(2 M)^{-1} \ln \left|m_{2}(0) / m_{1}(0)\right|$. Consideration of cases shows that this value of $t$ is positive iff $m_{1}(0)<0<m_{2}(0)$.

The event when $l_{k-1}=l_{k}=0$ is called a triple collision, since three particles come together at one point. The absence of triple collisions in the $\mathrm{CH}$ equation is a nontrivial result $[1,7]$, but for the linearly forced Burgers equation it is much simpler. (Note, however, that triple collisions are possible for discontinuous piecewise linear solution; see the examples at the end of the paper.) 
Theorem 4. Collisions occuring in continuous piecewise linear solutions of the linearly forced Burgers equation (1.2) cannot be triple collisions.

Proof. A triple collision would occur if $l_{k-1}\left(t_{0}\right)=0=l_{k}\left(t_{0}\right)$ for some $t_{0}$, which amounts to $F_{k-1}\left(t_{0}\right)=0=F_{k}\left(t_{0}\right)$ by (2.9). From the definition of $F_{k}$, it is obvious that this is impossible in the case $M=0$, since we are assuming $m_{k} \neq 0$. In the case $M \neq 0$, it is also impossible, although less obvious; $F_{k}\left(t_{0}\right)=0$ iff $t_{0}=\frac{1}{2 M} \log \frac{-\sum_{j>k} m_{j}(0)}{m_{k}(0)+\sum_{j<k} m_{j}(0)}$ and the quotient inside the logarithm is positive. So $F_{k}=F_{k-1}=0$ iff $\frac{m_{k}(0)+B}{A}=\frac{B}{m_{k}(0)+A}<0$, where $A=\sum_{j<k} m_{j}(0)$ and $B=\sum_{j>k} m_{j}(0)$, which requires that $\left(m_{k}+A\right)\left(m_{k}+B\right)=A B$, and hence $m_{k}\left(A+m_{k}+B\right)=0$. But this is ruled out by $m_{k}$ and $A+m_{k}+B=M$ both being nonzero.

We finish this section with the postponed proof of the main theorem.

Proof of Theorem 2. Assume to begin with that $x_{1}(0)<\cdots<x_{n}(0)$. Then (2.4) is equivalent to (2.1), and we can attack the problem by trying to find $x_{k}(t)$ and $m_{k}(t)$ such that the corresponding piecewise linear $u(x, t)$ given by (1.7) satisfies the PDE (2.2). The $x_{k}$ 's divide the real line into $n+1$ intervals which we number by $k=0, \ldots, n$. In each such interval $u$ takes the form $u(x, t)=$ $a_{k}(t) x+b_{k}(t)$. Inserting this into (2.2) yields $\dot{a}_{k}+a_{k}^{2}=M^{2}$ and $\dot{b}_{k}+b_{k} a_{k}=-M M_{+}$, from which (in the case $M \neq 0$ )

$$
a_{k}(t)=M \frac{a_{k}(0) \cosh (M t)+M \sinh (M t)}{a_{k}(0) \sinh (M t)+M \cosh (M t)}
$$

is found immediately, and by making an ansatz for $b_{k}$ with the same denominator as $a_{k}$ one also obtains

$$
b_{k}(t)=\frac{a_{k}(0) M_{+}(1-\cosh (M t))+M\left(b_{k}(0)-M_{+} \sinh (M t)\right)}{a_{k}(0) \sinh (M t)+M \cosh (M t)} .
$$

Now $x_{k}(t)$ and $m_{k}(t)$ are recovered from the relations $m_{k}=\frac{1}{2}\left(a_{k}-a_{k-1}\right)$ and $x_{k}=-\left(b_{k}-b_{k-1}\right) /\left(a_{k}-\right.$ $\left.a_{k-1}\right)$. Because of the algebraic nature of the formulas thus obtained, they satisfy the ODEs (2.4) identically, which shows that the assumption $x_{1}<\ldots<x_{n}$ is immaterial and can be removed. (This will be important later; see the comments after Theorem 8.) The simpler case $M=0$ (unforced Burgers) is entirely similar, except that

$$
a_{k}(t)=\frac{a_{k}(0)}{t a_{k}(0)+1}, \quad b_{k}(t)=\frac{b_{k}(0)}{t a_{k}(0)+1} .
$$

(The solution for $M=0$ can also be obtained by expanding $e^{ \pm M t}=1 \pm M t+O\left(M^{2}\right)$ in the solution for $M \neq 0$ and letting $M \rightarrow 0$.)

\section{Inverse spectral construction of solutions}

The Lax pair

$$
\begin{aligned}
-\partial_{x}^{3} \phi & =z m \phi, \\
\phi_{t} & =\left[z^{-1} \partial_{x}^{2}+c+u_{x}-u \partial_{x}\right] \phi,
\end{aligned}
$$


with $c$ an arbitrary constant, is compatible iff $m_{t}+m_{x} u+3 m u_{x}=0$ and $m_{x}=u_{x x x}$, under the assumption of sufficient smoothness needed to justify the cross-differentiation. In particular, it is compatible if $u$ evolves according to the derivative Burgers equation (1.1), which can be written as $m_{t}+m_{x} u+3 m u_{x}=0$ with $m=u_{x x}$. To obtain the linearly forced Burgers equation (1.2) from equation (1.1) the rule $\left(u^{2}\right)_{x}=2 u u_{x}$ is used. It is not obvious if all these formal calculations have any relevance to weak solutions, where the smoothness assumptions may be violated. To investigate this, let us say that (3.1) and (3.2) constitute a weak Lax pair if they are satisfied in the weak sense discussed in the introduction (thus $\phi$, like $u$, is a $\mathscr{D}^{\prime}(\mathbf{R})$-valued function of $t$, and the equations hold in the space of distributions $\mathscr{D}^{\prime}(\mathbf{R})$ ). Solutions $u$ of the form (1.7), $u=\sum m_{k}\left|x-x_{k}\right|$, do admit a weak Lax pair with $m=u_{x x}=2 \sum_{k=1}^{n} m_{k} \delta_{x_{k}}$, and $\phi$ is in this case a continuous function (in fact, it is piecewise a quadratic polynomial in $x$ with $t$-dependent coefficients). The product $m \phi$ in (3.1) is well-defined since the distribution $m$ can be multiplied by the continuous function $\phi$. We hope to treat weak Lax pairs in more depth in future papers. Here we just state a theorem which can be verified by careful use of the calculus of distributions.

Theorem 5. The following are equivalent conditions on a function $u$ of the form (1.7), $u=$ $\sum m_{k}\left|x-x_{k}\right|:$

1. $u$ is a weak solution to the linearly forced Burgers equation (1.2), and $\left\{x_{k}, m_{k}\right\}$ satisfy equations (2.1).

2. $u$ has a weak Lax pair (3.1), (3.2).

When $u=\sum m_{k}\left|x-x_{k}\right|$, a solution to equation (3.1) with the asymptotic condition $\phi(x, t ; z)=1$ for $x<x_{1}(t)$ will be consistent with the time evolution given by (3.2) provided that we choose the constant $c=-M$. Such a solution evaluated at $x>x_{n}(t)$ will take the form $\phi(x, t ; z)=A(t ; z) \frac{1}{2}(x-$ $\left.x_{n}\right)^{2}+B(t ; z)\left(x-x_{n}\right)+C(t ; z)$, where all three coefficients are polynomials in $z$, which, by equation (3.2), satisfy $\dot{A}=0, \dot{B}=M B$, and $\dot{C}=\frac{A}{z}+2 M C$ (see [10]). Thus it is consistent with equations (3.1) and (3.2) to impose the condition $A(t ; z)=0$, which together with $\phi=1$ for $x<x_{1}$ amounts to the boundary conditions $\phi_{x}(-\infty)=\phi_{x x}(-\infty)=\phi_{x x}(\infty)=0$. With these boundary conditions in place, the problem of solving the ODEs (2.1) becomes an isospectral deformation problem which can be solved if one knows how to solve the inverse problem for equation (3.1). This is exactly the inverse problem that was studied in [10] under the additional assumption that all $m_{k}(0)>0$. We now give a brief summary of results from that paper.

Theorem 6. The "Neumann-like discrete cubic string" boundary value problem

$$
-\partial_{x}^{3} \phi=z m \phi, \quad \phi_{x}(-\infty)=\phi_{x x}(-\infty)=\phi_{x x}(\infty)=0,
$$

where $m=2 \sum_{k=1}^{n} m_{k} \delta_{x_{k}}$ with all $m_{k}>0$, has a spectrum of the form $\left\{0=z_{0}<z_{1}<z_{2}<\cdots<\right.$ $\left.z_{n-1}\right\}$. There is a one-to-one (up to translations of $m$ along the $x$ axis) and onto spectral map $m \mapsto\{M, \mu\}$, where $M=\sum m_{k}>0$ and $\mu$ is a measure of the form $\mu=\sum_{j=1}^{n-1} b_{j} \delta_{z_{j}}$, with $b_{j}>0$ for $j=1, \ldots, n-1$ (see details in [10]). The inverse problem of recovering the discrete measure $m$ from $\{M, \mu\}$ has the explicit solution

$$
m_{n-k}=\frac{\mathscr{C}_{k} \mathscr{D}_{k}}{2 \mathscr{A}_{k+1} \mathscr{A}_{k}}, \quad x_{n-k+1}-x_{n-k} \equiv l_{n-k}=-\frac{2 \mathscr{A}_{k}}{\mathscr{D}_{k}^{\prime}} .
$$

in terms of determinants of bimoment matrices constructed out of the measure $\mu$ and the constant $M$ (see below). 
We recall the following definitions from [10]. Given a measure $\mu$, let

$$
\beta_{j}=\int z^{j} d \mu(z), \quad I_{i j}=I_{j i}=\iint \frac{z^{i} w^{j}}{z+w} d \mu(z) d \mu(w) .
$$

Let $\mathscr{A}_{0}=\mathscr{B}_{0}=\mathscr{C}_{0}=\mathscr{D}_{0}=1, \mathscr{A}_{1}=I_{00}+\frac{1}{2 M}, \mathscr{D}_{1}^{\prime}=\beta_{0}$, and for other values of $k$ let

$$
\begin{aligned}
& \mathscr{A}_{k}=\left|\begin{array}{cccc}
I_{00}+\frac{1}{2 M} & I_{01} & \cdots & I_{0, k-1} \\
I_{10} & I_{11} & \cdots & I_{1, k-1} \\
I_{20} & I_{21} & \cdots & I_{2, k-1} \\
\vdots & \vdots & & \vdots \\
I_{k-1,0} & I_{k-1,1} & \cdots & I_{k-1, k-1}
\end{array}\right|, \\
& \mathscr{B}_{k}=\left|\begin{array}{cccc}
I_{00} & I_{01} & \cdots & I_{0, k-1} \\
I_{10} & I_{11} & \cdots & I_{1, k-1} \\
\vdots & \vdots & & \vdots \\
I_{k-1,0} & I_{k-1,1} & \cdots & I_{k-1, k-1}
\end{array}\right|, \quad \mathscr{C}_{k}=\left|\begin{array}{cccc}
I_{11} & I_{12} & \cdots & I_{1 k} \\
I_{21} & I_{22} & \cdots & I_{2 k} \\
\vdots & \vdots & & \vdots \\
I_{k 1} & I_{k 2} & \cdots & I_{k k}
\end{array}\right|, \\
& \mathscr{D}_{k}=\left|\begin{array}{ccccc}
I_{10} & I_{11} & \cdots & I_{1, k-1} \\
I_{20} & I_{21} & \cdots & I_{2, k-1} \\
\vdots & \vdots & & \vdots \\
I_{k 0} & I_{k 1} & \cdots & I_{k, k-1}
\end{array}\right|, \quad \mathscr{D}_{k}^{\prime}=\left|\begin{array}{cccc}
\beta_{10} & \cdots & I_{1, k-2} \\
\beta_{1} & I_{20} & \cdots & I_{2, k-2} \\
\vdots & \vdots & & \vdots \\
\beta_{k-1} & I_{k 0} & \cdots & I_{k, k-2}
\end{array}\right| .
\end{aligned}
$$

In all these cases, the index $k$ agrees with the size $k \times k$ of the determinant. Note that $\mathscr{A}_{k}=$ $\mathscr{B}_{k}+\frac{1}{2 M} \mathscr{C}_{k-1}$ for $k \geq 1$.

Let us analyze the formula (3.3) for $l_{k}$ in order to compare it with (2.9) obtained earlier. First, (3.2) implies that the linearly forced Burgers equation induces a very simple evolution of the measure $\mu$, namely $\mu(z ; t)=e^{M t} \mu(z ; 0)$. Because of this it is easy to factor out the time dependence from all the determinants involved in (3.3). This elementary exercise leads to $l_{k}(t)=l_{k}(0) F_{k}(t)$, where

$$
F_{k}(t)=\frac{\mathscr{B}_{n-k}(0) e^{M t}+\frac{1}{2 M} C_{n-k-1}(0) e^{-M t}}{\mathscr{B}_{n-k}(0)+\frac{1}{2 M} C_{n-k-1}(0)} .
$$

This is in full agreement with (2.6) and (2.9). The formula for $m_{k}$ can be checked in a similar way.

\section{Discontinuous piecewise linear solutions}

Theorem 7. The discontinuous piecewise linear ansatz (1.9), $u=\sum\left(m_{k}\left|x-x_{k}\right|-s_{k} \operatorname{sgn}\left(x-x_{k}\right)\right)$, is a weak solution of the linearly forced inviscid Burgers equation (1.2) if and only if

$$
\begin{aligned}
\dot{x}_{k} & =\sum_{i=1}^{n}\left(m_{i}\left|x_{k}-x_{i}\right|+s_{i} \operatorname{sgn}\left(x_{i}-x_{k}\right)\right), \\
\dot{m}_{k} & =2 m_{k} \sum_{i=1}^{n} m_{i} \operatorname{sgn}\left(x_{i}-x_{k}\right), \quad \dot{s}_{k}=s_{k} \sum_{i=1}^{n} m_{i} \operatorname{sgn}\left(x_{i}-x_{k}\right),
\end{aligned}
$$

for $k=1, \ldots, n$. For this class of solutions, equation (1.2) takes the form

$$
u_{t}+\frac{1}{2}\left(u^{2}\right)_{x}=M^{2} x-M\left(M_{+}+S\right)
$$


with $M=\sum m_{k}$ and $M_{+}=\sum m_{k} x_{k}$ as before, and with $S=\sum s_{k}$. The quantities $M$ and $M_{+}+S$ are constants of motion, and so is $s_{k}^{2} / m_{k}$ for $k=1, \ldots, n$ (provided that $m_{k} \neq 0$ ).

Proof. We will repeatedly use the following distributional formula valid for an arbitrary piecewise differentiable function $f$ with points of discontinuity at $x_{1}, x_{2}, \ldots, x_{n}: f_{x}=\left\{f_{x}\right\}+\sum_{k=1}^{n}[f]_{k} \delta_{x_{k}}$, where $\left\{f_{x}\right\}$ means the ordinary derivative taken away from discontinuities and $[f]_{k}=f\left(x_{k}^{+}\right)-$ $f\left(x_{k}^{-}\right)$denotes the jump at $x_{k}$. Moreover, $u_{t}=\sum_{k}\left(\dot{m}_{k}\left|x-x_{k}\right|-\left(m_{k} \dot{x}_{k}+\dot{s}_{k}\right) \operatorname{sgn}\left(x-x_{k}\right)+2 s_{k} \dot{x}_{k} \delta_{x_{k}}\right)$. Now the left-hand side of (1.2), $u_{t}+\frac{1}{2}\left(u^{2}\right)_{x}$, must be a function since the right-hand side is a function; hence all Dirac deltas must cancel out. Similarly, there must be no Dirac deltas in the first or second $x$ derivatives of $u_{t}+\frac{1}{2}\left(u^{2}\right)_{x}$, These conditions give, in turn,

$$
\begin{aligned}
& 0=2 s_{k} \dot{x}_{k}+\frac{1}{2}\left[u^{2}\right]_{k}, \quad 0=-2\left(m_{k} \dot{x}_{k}+\dot{s}_{k}\right)+\frac{1}{2}\left[\left\{\left(u^{2}\right)_{x}\right\}\right]_{k}, \\
& 0=2 \dot{m}_{k}+\frac{1}{2}\left[\left\{\left(u^{2}\right)_{x x}\right\}\right]_{k} .
\end{aligned}
$$

An elementary computation of jumps for the case of piecewise continuous functions now produces (4.1). The coefficients of the forcing term in the PDE are identified from the smooth part of the term $\frac{1}{2}\left(u^{2}\right)_{x}$, while the constants of motion follow from (4.1).

Weak solutions to an initial value problem are usually not unique unless the PDE is supplemented with a so-called entropy condition that picks out the "physical" solution. In the case of the Burgers equation this condition requires $u$ to jump down, not up, at discontinuities. This is satisfied by the ansatz (1.9) if all shock strenght $s_{k}$ are nonnegative, so we will assume $s_{k} \geq 0$ from now on.

When considering the initial value problem for the ODEs (4.1) we can assume without loss of generality that $x_{1}(0)<x_{2}(0)<\ldots<x_{n}(0)$. Thus on a sufficiently small time interval we will still have $x_{1}(t)<x_{2}(t)<\ldots<x_{n}(t)$; in other words, the positions stay in the sector $X_{e}$ (see (2.3)). In $X_{e}$ the equations (4.1) can be written as

$$
\begin{aligned}
\dot{x}_{k} & =\sum_{i=1}^{n}\left(m_{i} \operatorname{sgn}(k-i)\left(x_{k}-x_{i}\right)-s_{i} \operatorname{sgn}(k-i)\right), \\
\dot{m}_{k} & =2 m_{k} \sum_{i=1}^{n} m_{i} \operatorname{sgn}(i-k), \quad \dot{s}_{k}=s_{k} \sum_{i=1}^{n} m_{i} \operatorname{sgn}(i-k),
\end{aligned}
$$

for $k=1, \ldots, n$. These equations can be solved explicitly, since the simple change of variables in the following theorem reduces them to the ODEs already solved in Theorem 2.

Theorem 8. If $\left\{x_{k}, m_{k}, s_{k}\right\}_{k=1}^{n}$ satisfy (4.4), if all $m_{k}(0) \neq 0$, and if

$$
y_{k}=x_{k}+s_{k} / m_{k},
$$

then $\left\{y_{k}, m_{k}\right\}_{k=1}^{n}$ satisfy (2.4) (with $y_{k}$ taking the place of $x_{k}$ everywhere).

Proof. Straightforward calculation.

Note that the initital values $y_{k}(0)$ will not necessarily be distinct or sorted in increasing order even though the $x_{k}(0)$ 's are, but this does not matter since the solution formulas of Theorem 2 are valid for any initial conditions. So Theorem 2 gives us $y_{k}(t)$ and $m_{k}(t)$ (note that $\sum m_{k} y_{k}=$ $\sum\left(m_{k} x_{k}+s_{k}\right)=M_{+}+S$ replaces $M_{+}$in the solution formula (2.5)), and we can then recover $s_{k}(t)$ from the fact that $s_{k}^{2} / m_{k}$ is constant for each $k$; this gives $s_{k}(t)=s_{k}(0) / \sqrt{F_{k-1}(t) F_{k}(t)}$, and allows 
us to also recover $x_{k}(t)=y_{k}(t)-s_{k}(t) / m_{k}(t)$. This solution $\left\{x_{k}, m_{k}, s_{k}\right\}$ to (4.4) is also the solution to (4.1), at least locally in some time interval around $t=0$ (so that the $x_{k}$ 's remain in the sector $\left.X_{e}\right)$.

For illustration, here is the general solution with shocks in the case $n=2$, when $M \neq 0, m_{1}(0) \neq$ $0, m_{2}(0) \neq 0$ :

$$
\begin{aligned}
m_{1}(t) & =\frac{m_{1}(0)}{F_{0}(t) F_{1}(t)}, \quad s_{1}(t)=\frac{s_{1}(0)}{\sqrt{F_{0}(t) F_{1}(t)}}, \\
m_{2}(t) & =\frac{m_{2}(0)}{F_{1}(t) F_{2}(t)}, \quad s_{2}(t)=\frac{s_{2}(0)}{\sqrt{F_{1}(t) F_{2}(t)}}, \\
x_{1}(t) & =\frac{M_{+}+S-K m_{2}(0) e^{-M t}}{M}-\frac{s_{1}(0)}{m_{1}(0)} \sqrt{F_{0}(t) F_{1}(t)}, \\
x_{2}(t) & =\frac{M_{+}+S+K m_{1}(0) e^{M t}}{M}-\frac{s_{2}(0)}{m_{2}(0)} \sqrt{F_{1}(t) F_{2}(t)}, \\
F_{0}(t) & =e^{-M t}, \quad F_{1}(t)=\frac{m_{1}(0) e^{M t}+m_{2}(0) e^{-M t}}{M}, \quad F_{2}(t)=e^{M t}, \\
K & =x_{2}(0)-x_{1}(0)+\frac{s_{2}(0)}{m_{2}(0)}-\frac{s_{1}(0)}{m_{1}(0)} .
\end{aligned}
$$

In the continuous case (2.1) we assumed all $m_{k} \neq 0$, but for (4.1) it does make sense to have $m_{k}=0$ provided that the corresponding $s_{k}$ is nonzero. If $m_{k}(0)=0$, then clearly $m_{k}(t)=0$ for all $t$, and the above solution procedure does not work. But this is easily fixed: just write down the general solution obtained for a nonzero initial value $m_{k}(0)=a$, and let $a \rightarrow 0$ there.

We will finish with a few examples that show how to deal with the solution when it hits the boundary of the sector $X_{e}$.

Example. A particular antisymmetric solution of (4.1) with $n=3$ is given by $-x_{1}=x_{3} \equiv \xi>0$, $x_{2}=0,-m_{1}=m_{3} \equiv \mu>0, m_{2}=0, s_{1}=s_{3}=0, s_{2} \equiv \sigma \geq 0$, where $\xi(t)=\xi(0) F(t)-\sigma(0) t$, $\mu(t)=\mu(0) / F(t), \sigma(t)=\sigma(0) / F(t)$, with $F(t)=1-2 \mu(0) t$. (These formulas are obtained either by reducing (4.1) to ODEs for $\xi, \mu, \sigma$ and solving them directly; or by assuming $m_{2}(0)=a \neq 0$, changing variables to $y_{1}=x_{1}, y_{2}=x_{2}+s_{2} / m_{2}, y_{3}=x_{3}$, writing down the general solution using Theorems 8 and 2, and letting $a \rightarrow 0$; or simply by noting that $M=0$ so that we are dealing with the unforced Burgers equation whose solution can be found in the textbook way using characteristics.) Since $M_{+}+S=2 \mu \xi+\sigma$ is constant in time, the wave profile (see Figure 1) is

$$
\begin{aligned}
u(x, t)= & -\mu(t)|x+\xi(t)|+\mu(t)|x-\xi(t)|-\sigma(t) \operatorname{sgn}(x) \\
= & \begin{cases}2 \mu(0) \xi(0)+\sigma(0), & x<-\xi(t), \\
-2 \mu(t) x+\sigma(t), & -\xi(t) \leq x<0, \\
0, & x=0, \\
-2 \mu(t) x-\sigma(t), & 0<x \leq \xi(t), \\
-(2 \mu(0) \xi(0)+\sigma(0)), & \xi(t)<x .\end{cases}
\end{aligned}
$$

If $\sigma(0)=0$ then this is a shockless solution (with $n=2$ really, since there is neither mass nor shock at the site $\left.x_{2}=0\right)$. It is defined until $\xi(t)=\xi(0) F(t)$ becomes zero at time $t_{\text {coll }}=(2 \mu(0))^{-1}$. Then $x_{1}$ and $x_{3}$ collide at $x=0$ while $m_{1}$ and $m_{3}$ blow up to $-\infty$ and $+\infty$, respectively. However, 

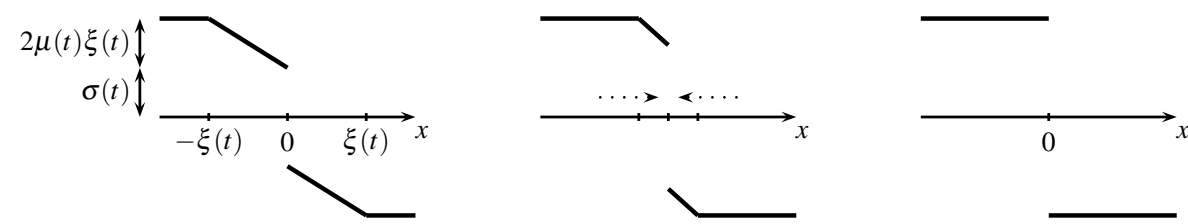

Figure 1: Left/middle: Wave profile $u(x, t)$ as given by (4.7) at two different times $t<t_{\text {coll }}$, with $\xi(t)$ decreasing towards zero at a constant rate. Right: Stationary profile after collision $\left(t \geq t_{\text {coll }}\right)$.

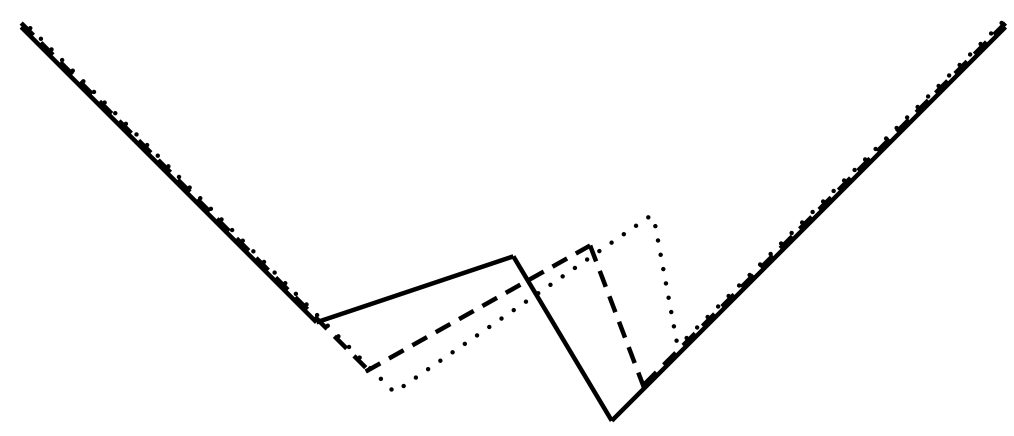

$x_{1}(0)=-2 \quad x_{2}(0)=0 \quad x_{3}(0)=1$

Figure 2: Solid: Continuous initial wave profile $u(x, 0)$. Dashed/dotted: $u(x, t)$ at times $t=\ln \frac{4}{3}$ and $t=\ln \frac{5}{3}$, respectively.

$u$ remains bounded, and tends to a shock profile: $u(x, t) \rightarrow-2 \mu(0) \xi(0) \operatorname{sgn}(x)$ as $t / t_{\text {coll }}$. This illustrates that shocks can form naturally even if they are not present in the initial wave profile. The profile will be stationary after the collision, because its continued evolution is given by the $n=1$ case of (4.1) $\left(\dot{x}_{1}=m_{1}, \dot{m}_{1}=\dot{s}_{1}=0\right)$ with $x_{1}=0, m_{1}=0, s_{1}=2 \mu(0) \xi(0)$. Consequently, $u(x, t)=-2 \mu(0) \xi(0) \operatorname{sgn}(x)$ for all $t \geq t_{\text {coll }}$.

If $\sigma(0)>0$ there is a shock waiting at the origin between the two approaching particles (as in Figure 1). The solution hits the boundary of the sector $X_{e}$ when $\xi(t)$ becomes zero at time $t_{\text {coll }}=(2 \mu(0)+\sigma(0) / \xi(0))^{-1}$. Then $x_{1}=x_{2}=x_{3}=0$, which illustrates that triple collisions may occur when shocks are present. Since the collision occurs earlier than in the shockless case, $F(t)$ has not yet reached zero at the time of collision; hence $m_{1}$ and $m_{3}$ do not blow up in this case. Again, $u$ tends to a stationary shock profile: $u(x, t)=-(2 \mu(0) \xi(0)+\sigma(0)) \operatorname{sgn}(x)$ for all $t \geq t_{\text {coll }}$.

Example. Consider now the shockless ODEs (2.1) with $n=3$ and initial data $m_{1}(0)=\frac{2}{3}, m_{2}(0)=$ -1 and $m_{3}(0)=\frac{4}{3}$, so that $M=1$. We assume $x_{1}(0)<x_{2}(0)<x_{3}(0)$ but leave them otherwise unspecified. Since $u= \pm\left(M x-M_{+}\right)$as $x \rightarrow \pm \infty$, and since the slope $u_{x}$ jumps by $2 m_{k}$ at each $x_{k}$, the initial profile $u(x, 0)$ consists of line segments with slope $-1, \frac{1}{3},-\frac{5}{3}$ and 1 , joined at the points $\left(x_{k}, u\left(x_{k}, 0\right)\right)$. Figure 2 illustrates this for the particular values $x_{1}(0)=-2, x_{2}(0)=0, x_{3}(0)=1$. Note that if the lines $u= \pm\left(M x-M_{+}\right)$to the left and to the right are continued, they intersect on the $x$ axis at the center of mass $x=M_{+} / M$ (=0 in the figure), which is a constant of motion.

Recall that $l_{1}=x_{2}-x_{1}$ and $l_{2}=x_{3}-x_{2}$. From (2.6) we obtain $F_{0}(t)=e^{-t}, F_{1}(t)=\frac{2}{3} e^{t}+$ 


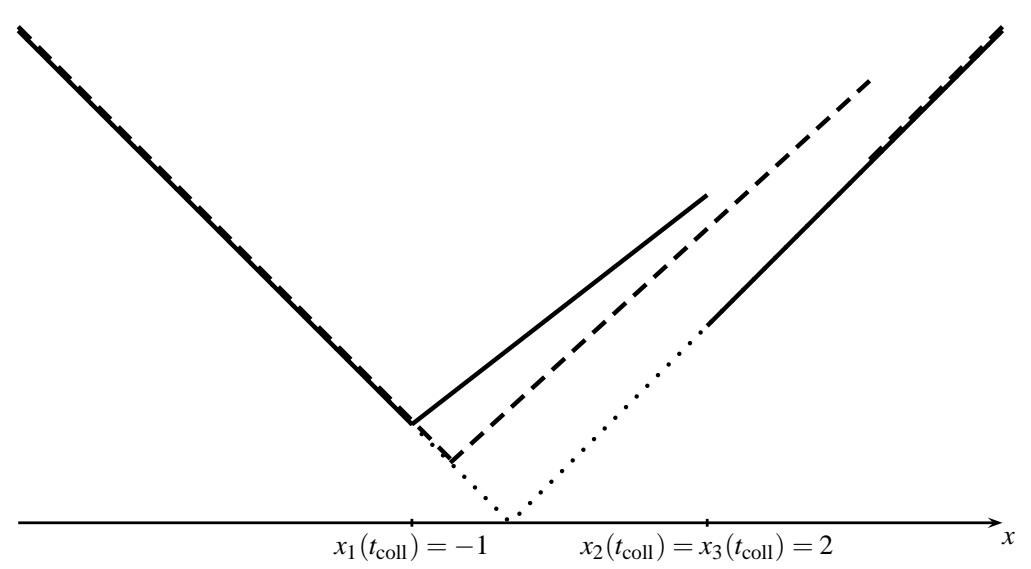

Figure 3: Solid: Discontinuous wave profile $u(x, t)$ formed at the instant of collision $t=t_{\text {coll }}=\ln 2$. Dashed: $u(x, t)$ at time $t=t_{\text {coll }}+\frac{1}{2}$. Dotted: No more collisions occur, and $u(x, t) \rightarrow|x|$ as $t \rightarrow+\infty$.

$\frac{1}{3} e^{-t}, F_{2}(t)=-\frac{1}{3} e^{t}+\frac{4}{3} e^{-t}$, and $F_{3}(t)=e^{t}$. Equations (2.5) and (2.9) give $x_{1}(t)=x_{1}(0)+(1-$ $\left.e^{-t}\right)\left(\frac{1}{3} l_{1}(0)+\frac{4}{3} l_{2}(0)\right), x_{2}(t)=x_{1}(t)+l_{1}(0) F_{1}(t)$, and $x_{3}(t)=x_{2}(t)+l_{2}(0) F_{2}(t)$. There is a collision between $x_{2}$ and $x_{3}$ when $F_{2}(t)$ becomes zero, which happens at time $t=t_{\text {coll }}=\ln 2$ when $e^{t}=2$. At that time we have $F_{0}=\frac{1}{2}, F_{1}=\frac{3}{2}, F_{2}=0, F_{3}=2$, hence by $(2.5) m_{1}=m_{1}(0) / F_{0} F_{1}=\frac{8}{9}$, $m_{2}=-\infty, m_{3}=+\infty$. As for the wave profile $u$, we have

$$
\begin{aligned}
u\left(x_{1}(t), t\right) & =m_{2} l_{1}+m_{3}\left(l_{1}+l_{2}\right)=\left(M-m_{1}\right) l_{1}+m_{3} l_{2} \\
& =\left(F_{1}-m_{1}(0) / F_{0}\right) l_{1}(0)+m_{3}(0) l_{2}(0) / F_{3} \\
& \rightarrow \frac{1}{6} l_{1}(0)+\frac{2}{3} l_{2}(0), \quad \text { as } t \nearrow t_{\mathrm{coll}},
\end{aligned}
$$

and

$$
\begin{aligned}
u\left(x_{2}(t), t\right)-u\left(x_{3}(t), t\right) & =\left(m_{1} l_{1}+m_{3} l_{2}\right)-\left(m_{1}\left(l_{1}+l_{2}\right)+m_{2} l_{2}\right) \\
& =\left(m_{3}-m_{1}-m_{2}\right) l_{2} \\
& =\left(m_{3}(0) / F_{3}-m_{1}(0) F_{2} / F_{0} F_{1}-m_{2}(0) / F_{1}\right) l_{2}(0) \\
& \rightarrow \frac{4}{3} l_{2}(0), \quad \text { as } t \nearrow t_{\text {coll }} .
\end{aligned}
$$

Thus the limiting wave profile at $t=t_{\text {coll }}$ consists of a line segment with slope -1 , joined to a line segment with slope $-1+2 \cdot \frac{8}{9}=\frac{7}{9}$ at $x=x_{1}\left(t_{\text {coll }}\right)=x_{1}(0)+\frac{1}{2}\left(\frac{1}{3} l_{1}(0)+\frac{4}{3} l_{2}(0)\right)$ and height $u=$ $\frac{1}{6} l_{1}(0)+\frac{2}{3} l_{2}(0)$; the profile jumps down by $\frac{4}{3} l_{2}(0)$ at $x=x_{2}\left(t_{\text {coll }}\right)=x_{3}\left(t_{\text {coll }}\right)=x_{1}\left(t_{\text {coll }}\right)+\frac{3}{2} l_{1}(0)$, and continues from there with slope 1 . See Figure 3.

The continued evolution of the profile for $t \geq t_{\text {coll }}$ is illustrated in Figure 3; it is given by the shock ODEs (4.1) with $n=2$, using a new set of variables whose initial values at $t=t_{\text {coll }}$ are $\tilde{x}_{1}=x_{1}\left(t_{\text {coll }}\right), \tilde{x}_{2}=x_{2}\left(t_{\text {coll }}\right), \tilde{m}_{1}=\frac{8}{9}, \tilde{m}_{2}=\frac{1}{9}, \tilde{s}_{1}=0$, and $\tilde{s}_{2}=\frac{2}{3} l_{2}(0)$. In terms of the new time variable $\tau=t-t_{\text {coll }} \geq 0$ one finds from the general solution (4.6) that, for example,

$$
\tilde{x}_{2}(\tau)-\tilde{x}_{1}(\tau)=\left(\tilde{x}_{2}(0)-\tilde{x}_{1}(0)+\frac{\tilde{s}_{2}(0)}{\tilde{m}_{2}(0)}\right) \tilde{F}_{1}(\tau)-\frac{\tilde{s}_{2}(0)}{\tilde{m}_{2}(0)} \sqrt{\tilde{F}_{1}(\tau) \tilde{F}_{2}(\tau)},
$$

where $\tilde{F}_{1}(\tau)=\frac{8}{9} e^{\tau}+\frac{1}{9} e^{-\tau}$ and $\tilde{F}_{2}(\tau)=e^{\tau}$. Writing this expression as $\tilde{x}_{2}-\tilde{x}_{1}=(A+B) \tilde{F}_{1}-$ $B \sqrt{\tilde{F}_{1} \tilde{F}_{2}}$, we see that it is zero if $F_{1}(\tau)=0$, which can never happen, or if $(A+B)^{2} F_{1}=B^{2} F_{2}$, 
which is the same as $e^{-2 \tau}=9((A+B) / B)^{2}-8$ that can't happen either since the right-hand side is $>1$ and the left-hand side is $\leq 1$ for $\tau \geq 0$. The conclusion is that, in this example, $\tilde{x}_{2}(\tau)-\tilde{x}_{1}(\tau)$ remains positive for all $\tau>0$, so there are no more collisions. Instead, as $\tau$ (or $t$ ) $\rightarrow+\infty$, we have $\tilde{x}_{1} \rightarrow 0, \tilde{x}_{2} \rightarrow+\infty, \tilde{m}_{1} \rightarrow M, \tilde{m}_{2} \rightarrow 0$, and $\tilde{s}_{2} \rightarrow 0$. Thus, $u(x, t)$ approaches the limiting wave profile $u(x,+\infty)=|x|$.

Acknowledgments. HL's participation in NEEDS 2007 was supported by a travel grant from the Knut and Alice Wallenberg Foundation. JS is supported by the Natural Sciences and Engineering Research Council of Canada (NSERC), Grant No. 138591-04.

\section{References}

[1] Beals R, Sattinger D and Szmigielski J, Multipeakons and the classical moment problem, Advances in Mathematics 154 (2000), 229-257.

[2] Beals R, Sattinger D H and Szmigielski J, Inverse scattering solutions of the Hunter-Saxton equation, Appl. Anal. 78 (2001), 255-269.

[3] Bressan A and Constantin A, Global conservative solutions of the Camassa-Holm equation, Arch. Ration. Mech. Anal. 183 (2007), 215-239.

[4] Camassa R and Holm D D, An integrable shallow water equation with peaked solitons, Phys. Rev. Lett. 71 (1993), 1661-1664.

[5] Degasperis A, Holm D D and Hone A N W, A new integrable equation with peakon solutions, Theoretical and Mathematical Physics 133 (2002), 1463-1474, nlin.SI/0205023.

[6] Degasperis A and Procesi M, Asymptotic integrability, In A Degasperis and G Gaeta, eds., Symmetry and perturbation theory (Rome, 1998), 23-37. World Scientific Publishing, River Edge, NJ (1999).

[7] Holden H and Raynaud X, Global conservative multipeakon solutions of the Camassa-Holm equation, J. Hyperbolic Differ. Equ. 4 (2007), 39-64.

[8] Hunter J K and Saxton R, Dynamics of director fields, SIAM J. Appl. Math. 51 (1991), 14981521.

[9] HUNTER J K and ZHENG Y X, On a completely integrable nonlinear hyperbolic variational equation, Phys. D 79 (1994), 361-386.

[10] Kohlenberg J, Lundmark H and Szmigielski J, The inverse spectral problem for the discrete cubic string, Inverse Problems 23 (2007), 99-121.

[11] Lundmark H, Formation and dynamics of shock waves in the Degasperis-Procesi equation, $J$. Nonlinear Sci. 17 (2007), 169-198.

[12] Lundmark H and Szmigielski J, Multi-peakon solutions of the Degasperis-Procesi equation, Inverse Problems 19 (2003), 1241-1245.

[13] Lundmark H and SzMigielski J, Degasperis-Procesi peakons and the discrete cubic string, IMRP Int. Math. Res. Pap. 2005 (2005), 53-116. 